\title{
Gelsolin levels in patients with bronchiolitis
}

Feyza Hüsrevoğlu-Esen ${ }^{1}$, Yasemin Altuner-Torun², Çiğdem Karakükçü ${ }^{3}$, Seçil Köse², Ahmet Şehriyaroğlu², Dilek Kafadar ${ }^{2}$, Aydın Esen², Mehmet Köse ${ }^{4}$

${ }^{1}$ Division of Pediatric Emergency Medicine, ${ }^{4}$ Division of Pediatric Pulmonology, Department of Pediatrics, Erciyes University Faculty of Medicine; Departments of ${ }^{2}$ Pediatrics, ${ }^{3}$ Biochemistry, Kayseri Education and Research Hospital Kayseri, Turkey. E-mail: feyzahusrevoglu@hotmail.com

Received: 18th August 2017, Revised: 30th September 2017, Accepted: 30th October 2017

SUMMARY: Hüsrevoğlu-Esen F. Altuner-Torun Y, Karakükçü Ç, Köse S, Şehriyaroğlu A, Kafadar D, Esen A, Köse M. Gelsolin levels in patients with bronchiolitis. Turk J Pediatr 2018; 60: 286-289.

Bronchiolitis is the leading cause of hospitalization in infants. Biomarkers can show severity of the disease and help in clinical management. In this study, the aim was to determine the clinical predictiveness of plasma gelsolin levels (pGSN) in acute bronchiolitis.

From December 2013 to May 2014, 52 patients with bronchiolitis (aged <24 months) were included in this study. Baseline clinical characteristic, complete blood count, C-Reactive Protein, plasma gelsolin levels, chest X-rays were obtained in all patients. The patients were divided into three groups as mild, moderate and severe based on clinical findings.

There was no significant difference in pGSN levels between the control group and 3 study groups according to their clinical scores such as mild, moderate and severe bronchiolitis ( $p>0.05)$.

Recent studies reported that pGSN levels can be used as a biomarker in sepsis, inflammation and injuries. In this study, we have demonstrated that pGSN level is not a predictive biomarker of bronchiolitis and its severity. Hence, we hypothesized that pGSN levels can be used in bacterial infections rather than viral infections as a biomarker.

Key words: acute bronchiolitis, gelsolin, biomarker.

Acute bronchiolitis in children is characterized by a viral upper respiratory prodrome followed by increased respiratory effort and wheezing; while the most common etiologic agent is Respiratory Syncytial Virus (RSV). It is caused by bronchial obstruction because of edema, mucus accumulation and cell damage after virus invasion to the small airways. Depending on inflammation, airway epithelial necrosis occurs, epithelium is replaced with nonciliated epithelium, consequently, secretion to the larger proximal airways is reduced and airway obstruction develops. The diagnosis of bronchiolitis is mostly clinical and usually does not require investigation. Guidelines have classified bronchiolitis into: mild, moderate and severe according to clinical findings. ${ }^{1}$

Plasma gelsolin (pGSN) scavenges actin filaments released from cells during inflammation and injury. 2,3 It was reported that of recombinant human pGSN infusion at appropriate doses can diminish evolving injury or reduce mortality rates in animal models of hyperoxia, burns, and sepsis. ${ }^{4-6}$

Tissue injuries in various organs and diseases lead to prolonged reductions in pGSN levels, which precedes and predicts the development of many of the morbidities experienced by these high risk patients, including acute respiratory distress syndrome, acute lung injury, multiple organ dysfunction syndrome, and sepsis. In such patients, the degree of the reduction in pGSN levels has an inverse relationship with the duration of assisted ventilation, length of intensive care unit stay, overall length of hospital stay, and death. ${ }^{7-9}$

Although bronchiolitis is a clinical entity, there is no known biomarker that predicts 
the prognosis or survival. The aim of this study was to determine whether pGSN level is associated with viral infections especially in acute bronchiolitis.

\section{Material and Methods \\ Subjects}

This prospective study was conducted between December 1st, 2013 and May 1st, 2014 at Kayseri Training and Research Hospital. The study included 52 patients with acute bronchiolitis and 28 healthy children. Inclusion criteria were as follows: aged between 1 and 24 months, a history of preceding viral upper respiratory infection followed by wheezing and crackles on auscultation, first wheezing episode, and clinical severity score (CSS) $\geq$ 4. Bronchiolitis was diagnosed on clinical findings. The clinical severity score (CSS) was previously described by Wang et al. ${ }^{10}$ in Table I. Exclusion criteria were as follows: chronic cardiopulmonary or neurological disease, premature birth, birth weight $<2500$ $\mathrm{g}$, history of recurrent wheezing episodes, proven immune deficiency, age of $<1$ month old or $>2$ years, proven or suspected acute bacterial infection, previous treatment with bronchodilators or corticosteroids, symptoms $>7$ days, consolidation or atelectasis on a chest roentgenogram.

The control group comprised 28 healthy children (with similar age/gender) presented to Pediatric General Policlinics for routine health control or vaccination. pGSN level was studied only at baseline in the control group.

The parents of all patients and controls were informed about the study objectives and written informed consent was obtained before inclusion in the study. The ethical aspects were respected and the research was approved by the Ethics and Research Committee of Erciyes University.

\section{Evaluation of pGSN Levels}

All plasma samples were frozen at $-20^{\circ} \mathrm{C}$ until assays. Plasma gelsolin levels were measured by a quantitative sandwich enzyme immunoassay method by an ELISA kit (Biomatic, PGSN USA). A micro-plate precoated by an antibody specific for soluble human pGSN was used. Standards and samples were pipetted into the wells and any soluble human pGSN present was bound by the immobilized antibody. After washing away any unbound substances, a biotinylated antibody specific for soluble human pGSN was added to the wells. Following a wash to remove any unbound antibody reagent, a Streptavidin HRP conjugate was added to each well. After washing away any unbound enzyme, a substrate solution was added to each of the wells and color develops in proportion to the amount of soluble pGSN bound in the initial step. The color development was stopped and the intensity of the color was measured at 450 $\mathrm{nm}$. The concentration of pGSN in samples was determined by comparing the O.D. of the samples to the standard curve. Results were expressed as $\mathrm{ng} / \mathrm{ml}$.

\section{Statistical analysis}

All statistical analyses were performed using the IBM SPSS Statictics 22.0 package program (IBM Corp, Armonk, New York, USA). Data are expressed either as frequencies or median (min-max). Shapiro-Wilk's test was used and a histogram and q-q plot were examined to assess the normality. A two-sided Kruskal Wallis test was applied to compare the differences between groups for continuous variables. A p values less than 0.05 were considered statistically significant.

Table I. Clinical Severity Score.

\begin{tabular}{|c|c|c|c|}
\hline & 1 point & 2 point & 3 point \\
\hline $\begin{array}{l}\text { Respiratory rate } \\
\text { Breaths/min }\end{array}$ & $31-45$ & $46-60$ & $>60$ \\
\hline Wheezing & $\begin{array}{l}\text { At terminal } \\
\text { expiration using } \\
\text { a stethoscope }\end{array}$ & $\begin{array}{l}\text { During the entire expiration } \\
\text { or audible on expiration } \\
\text { without stethoscope }\end{array}$ & $\begin{array}{l}\text { Inspiratory and expiratory wheezing } \\
\text { without stethoscope }\end{array}$ \\
\hline Retraction & intercostal & Tracheo sternal & Severe retraction with nasal flaring \\
\hline General Condition & normal & stable & $\begin{array}{c}\text { Irritability, lethargy and poor } \\
\text { appetite }\end{array}$ \\
\hline
\end{tabular}


Table II. Group's Demographic Variables and Clinical Findings

\begin{tabular}{lcccc}
\hline & Mild & Moderate & Severe & p value \\
\hline Age (month) & 20 & 19 & 13 & 0.41 \\
Gender & M:12 & M:8 & M:7 & 0.90 \\
& F:8 & F:11 & F: 6 & 0.85 \\
Hospital stay (hour) & 2 & 74.6 & 101.3 & 0.07 \\
$\mathrm{O}_{2}$ requirement (hour) & 0 & 12 & 34.2 & 0.09 \\
Ventolin dose (n) & 4.7 & 20.9 & 27,3 & 0.09 \\
WBC & 7780 & 8276 & 9540 & 0.25 \\
CRP (mg/L) & 3.25 & 13.5 & 11.9 & 0.50 \\
Steroid taken (n) & 0 & 10 & 10 & 0.10 \\
Antibiotic taken (n) & 1 & 9 & 5 & 0.96 \\
X-ray finding (n) & 0 & 1 & 4 & 0.56 \\
RSV inf. (n) & 20 & 19 & 13 & 0.43 \\
\hline
\end{tabular}

WBC: white blood count, CRP: C-reactive protein, RSV: respiratury syncytial virus

\section{Results}

Fifty-two patients were enrolled in the study. There were 20 patients in mild group, 20 patients in moderate and 12 patients in severe group according to CSS.

Five patients had a chest X-ray finding (diffuse interstitial consolidation), while 15 patients received antibiotic therapy, and 20 patients received steroid therapy (all of the severe group and 8 from the moderate group). All the patients had supportive therapy such as hydration, oxygen and salbutamol nebules if indicated.

There were no significant differences between pGSN levels and patient's age $(\mathrm{p}=0.41)$, gender $(p=0.90,0.85)$, hospitalization time $(\mathrm{p}=0.07)$, hours of $\mathrm{O}_{2}$ therapy $(\mathrm{p}=0.09)$, doses of ventolin $(\mathrm{p}=0.09)$, steroid requirement $(\mathrm{p}=0.10)$, antibiotic taken (0.96), CRP levels $(p=0.50)$, white blood counts $(p=0.25)$, chest X-ray findings $(p=0.56)$, having RSV or not $(p=0.43)$ (Table II). All the patients had RSV infections that were detect from nasopharyngeal aspirates.

The control group included 28 healthy children with a mean age of 20 months old. There were no significant differences in pGSN levels between the control group and three patient groups according to clinical scores such as mild, moderate and severe bronchiolitis $(p=0.930)$. Median pGSN levels at the time of diagnosis was $142.17 \mathrm{ng} / \mathrm{ml}$ in the mild group; 143.38 $\mathrm{ng} / \mathrm{ml}$ in the moderate group, $139.0 \mathrm{ng} / \mathrm{ml}$ in the severe group and $140.97 \mathrm{ng} / \mathrm{ml}$ in the control group.

\section{Discussion}

Bronchiolitis is the most common cause of hospitalization among infants during the first 12 months of life. Approximately 100000 bronchiolitis admissions occur annually in the United States at an estimated cost of $\$ 1.73$ billion. ${ }^{11}$ RSV alone is estimated to have caused 66000 to 199000 deaths among children younger than 5 years of age. ${ }^{12}$ Despite the severity of the disease, it is difficult to define the best possible curative therapy and the only known therapy is often supportive. Research on new biomarkers could help us to determine the severity of bronchiolitis, prognosis, and need for intensive care unit.

Plasma pGSN is an important member of the extracellular actin scavenger system, which binds to actin micro-filaments, released into circulation because of cell death. When actin levels increase in micro circulation, pGSN levels decrease. In bronchiolitis, there is a viral inoculation of respiratory epithelium which leads to inflammation of small airways and sloughing off the epithelium causes varying degrees of bronchial obstruction. In this regard, we planned this study around the question of whether this epithelial damage triggers the scavenger system and hence affects pGSN level.

This is the first study on pGSN levels in patients with acute bronchiolitis. In this study, 
there was no statistical difference in pGSN levels between control group and the patients with mild, moderate and severe groups of bronchiolitis. Reduced pGSN levels have been reported in acute respiratory distress syndrome, sepsis, trauma and septic shock. ${ }^{6}$ Cohen et al. ${ }^{13}$ obtained evidence indicating that exogenous pGSN might reduce morbidity from sepsis in a rat model. Kose et al. ${ }^{14}$ reported that pGSN levels decreased in newborn sepsis. Kulakowska et al. ${ }^{15}$ demonstrated that depletion of plasma pGSN in patients with tick-borne encephalitis and Lyme neuroborreliosis. A recent study showed that pGSN levels are lower in preterm infants with sepsis than those of control group, and pGSN levels increased after recovery in patient with sepsis. ${ }^{16}$ Although pGSN levels decreased in septic patients, in our study there is no statistically significant difference between three clinic scores of patients with bronchiolitis. Thus, we considered that pGSN levels are changed more often in bacterial infections such as sepsis, than viral infections.

Our study had some limitations. Firstly, the number of patients were limited. Secondly, we could not detect any other viral agents except for RSV because of the insufficiency of our microbiology laboratory. Upcoming information from clinical studies will highlight the specific viral infections and pGSN level correlations with a large number of patients.

In conclusion, pGSN levels did not change in our study. Therefore, we considered that pGSN is a biomarker of bacterial infections and its level does not change in viral infections. In addition, further studies involving viral infections and pGSN would provide a better understanding of the pGSN as a biomarker in bacterial infections and allow the development of new treatment modalities.

\section{Acknowledgements}

All authors contributed extensively and equally to this work. This research received funding from Kayseri Training and Research Hospital

\section{REFERENCES}

1. Ravaglia C, Poletti V. Recent advances in the management of acute bronchiolitis. F1000Prime Rep 2014; 6: 103.

2. Lind SE, Smith DB, Janmey PA, Stossel TP. Role of plasma gelsolin and the vitamin D-binding protein in clearing actin from the circulation. J Clin Invest 1986; 78: $736-742$
3. Vasconcellos CA, Allen PG, Wohl ME, Drazen JM, Janmey PA, Stossel TP. Reduction in viscosity of cystic fibrosis sputum in vitro by gelsolin. Science 1994; 263 : 969-971.

4. Suhler E, Lin W, Yin HL, Lee WM. Decreased plasma gelsolin concentrations in acute liver failure, myocardial infarction, septic shock, and myonecrosis. Crit Care Med 1997; 25: 594-598.

5. Mounzer KC, Moncure M, Smith YR, Dinubile MJ Relationship of admission plasma gelsolin levels to clinical outcomes in patients after major trauma. Am J Respir Crit Care Med 1999; 160(5 Pt 1): 1673-1681.

6. Peddada N, Sagar A, Ashish GR, Garg R. Plasma gelsolin: A general prognostic marker of health. Med Hypotheses 2012; 78: 203-210.

7. Lee PS, Waxman AB, Cotich KL, Chung SW, Perrella MA, Stossel TP. Plasma gelsolin is a marker and therapeutic agent in animal sepsis. Crit Care Med 2007; 35: 849-855.

8. Christofidou-Solomidou M, Scherpereel A, Solomides $\mathrm{CC}$, et al. Recombinant plasma gelsolin diminishes the acute inflammatory response to hyperoxia in mice. J Investig Med 2002; 50: 54-60.

9. Rothenbach PA, Dahl B, Schwartz JJ, et al. Recombinant plasma gelsolin infusion attenuates burn-induced pulmonary microvascular dysfunction. J Appl Physiol (1985) 2004; 96: 25-31.

10. Wang EE, Milner RA, Navas L, Maj H. Observer agreement for respiratory signs and oximetry in infants hospitalized with lower respiratory infections. Am Rev Respir Dis 1992; 145: 106-109.

11. Hasegawa K, Tsugawa Y, Brown DF, Mansbach JM, Camargo CA Jr. Trends in bronchiolitis hospitalizations in the United States, 2000-2009. Pediatrics 2013;132: 28-36.

12. Nair H, Nokes DJ, Gessner BD, et al. Global burden of acute lower respiratory infections due to respiratory syncytial virus in young children: A systematic review and meta-analysis. Lancet 2010; 375: 1545-1555.

13. Cohen TS, Bucki R, Byfield FJ, et al. Therapeutic potential of plasma gelsolin administration in a rat model of sepsis. Cytokine 2011; 54: 235-238.

14. Kose M, Elmas T, Gokahmetoglu S, et al. Predictive value of gelsolin for the outcomes of preterm neonates: A pilot study. Pediatr Int 2014; 56: 856-859.

15. Kulakowska A, Zajkowska JM, Ciccarelli NJ, Mroczko B, Drozdowski W, Bucki R. Depletion of plasma gelsolin in patients with tick-borne encephalitis and Lyme neuroborreliosis. Neurodegener Dis 2011; 8: 375-380.

16. Halis H, Gunes T, Korkut S, et al. In the diagnosis of neonatal sepsis importance of gelsolin and relationship with mortality and morbidity. Med Hypotheses 2016; 94: 77-80. 